The authors conclude that antimalarial agents might have a protective effect against neoplasia, although this hypothesis requires further investigation.

Original article Ruiz-Irastorza G et al. (2007) Antimalarials may influence the risk of malignancy in systemic lupus erythematosus. Ann Rheum Dis 66: 815-817

\section{Anti-TNF therapy does not increase the risk of serious bacterial infection in RA patients}

Anti-tumor necrosis factor (anti-TNF) agents, such as etanercept, infliximab and adalimumab, are widely prescribed for patients with arthritic diseases. There are concerns, however, regarding the short-term and long-term risks of bacterial infection associated with anti-TNF therapy. Schneeweiss et al. performed a cohort study in elderly patients with rheumatoid arthritis (RA) to assess the risk of serious, hospitalizing infections associated with anti-TNF and other DMARD therapies.

The study included 15,597 patients with $R A$ aged $\geq 65$ years (mean 76.5 years) who were Medicare beneficiaries and who were also enrolled in the Pharmaceutical Assistance Contract for the Elderly (PACE) program of Pennsylvania. The patients initiated use of methotrexate $(n=1,900)$, anti-TNF therapy $(n=469)$, other cytotoxic DMARDs $(n=654)$, noncytotoxic DMARDs $(n=1,957)$, or glucocorticoids $(n=10,617)$ between January 1995 and December 2003.

Length of follow-up was $0.20-1.29$ years. The overall mean incidence of serious bacterial infections that required hospitalization (e.g. pneumonia, septicemia and osteomyelitis) was 2.2 per 100 patient-years. Glucocorticoid use was associated with a twofold increase in rate of serious infections compared with methotrexate use (adjusted rate ratio $2.1,95 \% \mathrm{Cl} 1.5-3.1$ ), with a significant positive dose-dependent trend $(P<0.0001)$. There was no significant increase in serious infection rate associated with anti-TNF therapy or use of other DMARDs compared with methotrexate.

The authors conclude that anti-TNF therapy does not increase the risk of serious bacterial infection in elderly patients with RA compared with methotrexate, and that their findings regarding glucocorticoids support those from previous studies.

Original article Schneeweiss S et al. (2007) Anti-tumor necrosis factor $\alpha$ therapy and the risk of serious bacterial infections in elderly patients with rheumatoid arthritis. Arthritis Rheum 56: 1754-1764

\section{Does smoking influence anti-CCP-positive rheumatoid arthritis?}

Smoking has previously been shown to be associated with rheumatoid factor (RF)-positive rheumatoid arthritis (RA). A European study found an association between smoking, anticyclic citrullinated peptide (anti-CCP) and shared epitope alleles; however, a US study that investigated this link found only weak association between smoking and anti-CCP RA.

In a case-only analysis, Lee et al. evaluated the link between smoking, the shared epitope, and anti-CCP RA in 3 large patient populations: the North American RA Consortium (NARAC), the National Inception Cohort of RA patients, and the Study of New-Onset RA (SONORA). The researchers found a significant association between smoking and the presence of antiCCP in NARAC and the Inception Cohort (odds ratios 1.38 and 1.41, respectively), but not in SONORA. However, smoking was not found to significantly influence the association between the shared epitope and anti-CCP positivity. Regardless of smoking status, the shared epitope was found to have a dose-dependent effect on anti-CCP levels, with homozygotes for the epitope more likely to be anti-CCP-positive than heterozygotes. An association between heavy smoking ( $\geq 20$ pack-years) and anti-CCP positivity was found in the Inception Cohort, but this link could not be confirmed in the other cohorts because of data limitations.

The authors state that they are unable to confirm an interaction between the shared epitope, smoking and anti-CCP RA in North American patients, and overall the association between smoking and CCP-positive RA is weaker than that found in the Swedish population. They suggest that environmental factors other than, or in addition to smoking, might predominate in the development of RA in North America.

Original article Lee HS et al. (2007) Interaction between smoking, the shared epitope, and anti-cyclic citrullinated peptide. Arthritis Rheum 56: 1745-1753 